\title{
Complete Reversibility of Physiological Coronary Vascular Abnormalities in Hypertrophied Hearts Produced by Pressure Overload in the Rat
}

\author{
Shogen Isoyama, Nobuhiko Ito, Masao Kuroha, and Tamotsu Takishima \\ First Department of Internal Medicine, Tohoku University School of Medicine, Sendai 980, Japan
}

\begin{abstract}
Using an experimental model of ascending aortic banding in the rat, we examined whether coronary circulation abnormalities in hypertrophied hearts are reversible after debanding. 4-wk banding produced significant increases in in vivo left ventricular (LV) pressure (194 \pm 13 vs. $114 \pm 9 \mathrm{mmHg}$ in shamoperated controls) and LV dry wt/body wt (48 $\pm 5 \%$ above controls). In isolated hearts perfused with Krebs-Henseleit buffer, coronary flow rate (CFR) was estimated under nonworking conditions. During maximal vasodilation after 1 min-ischemia, CFR at a coronary perfusion pressure (CPP) of $100 \mathrm{mmHg}$ and CFR/myocardidial mass at CPPs of 100 and $150 \mathrm{mmHg}$ decreased significantly $(72 \pm 5 \% ; 53 \pm 4$ and $61 \pm 4 \%$ of controls). 1 or 4 wk after debanding, LV systolic pressures were similar to control values, and the degree of myocardial hypertrophy decreased to levels $23 \pm 6(P<0.01)$ and $11 \pm 6 \%(P<0.01)$ above their control values, respectively. At 1 wk there was no significant increase in CFR/myocardial mass, compared to values in the banded group (67 \pm 8 vs. $53 \pm 4 \%$ of controls at $100 \mathrm{mmHg}$ and $67 \pm 9$ vs. $61 \pm 4 \%$ at $150 \mathrm{mmHg}$ of CPP). At 4 wk, CFR and the ratio had increased toward normal. Thus, decreased coronary perfusion in hypertrophied hearts is completely reversible.
\end{abstract}

\section{Introduction}

Hemodynamic overload causes cardiac muscle hypertrophy, as an adaptative process of the heart (1). In hypertrophied hearts, abnormalities in coronary circulation such as decreased coronary blood flow per unit muscle mass (2), increased coronary vascular resistance during maximal vasodilation (3-9), impaired autoregulation (10), changes in transmural blood flow distribution $(3,9-13)$, or decreased coronary flow reserve $(6-9,14-22)$ have been reported in clinical and animal studies. If the hemodynamic load is normalized, cardiac muscle hypertrophy regresses in patients and animals (1, 23-31). However, it has not been determined whether the coronary circulation abnormalities in hypertrophied hearts are reversible after normalization of the hemodynamic load, as in the regression of cardiac muscle hypertrophy.

The abnormalities in coronary circulation might be caused indirectly by the presence of cardiac muscle hypertrophy sur-

Address reprint requests to Dr. Takishima, First Department of Internal Medicine, Tohoku University School of Medicine, 1-1 Seiryomachi, Sendai 980, Japan.

Received for publication 28 September 1988 and in revised form 19 January 1989.

J. Clin. Invest.

(C) The American Society for Clinical Investigation, Inc. 0021-9738/89/07/0288/07 \$2.00

Volume 84, July 1989, 288-294 rounding the coronary vascular system per se (9), directly by coronary vascular changes produced by coronary arterial hypertension with or without significant cardiac muscle hypertrophy $(4,6)$, or by both. Therefore, the time course of normalization of physiological coronary vascular abnormalities in hypertrophied hearts in response to pressure overload with coronary arterial hypertension may differ from that in the regression process of myocardial hypertrophy, even if the coronary circulation abnormalities are reversible.

In this study, we used a rat model of ascending aortic banding, i.e., pressure overload to the left ventricle and high coronary arterial pressure, and examined the regression process in coronary circulation abnormalities and cardiac muscle hypertrophy after debanding. To minimize the effect of mechanical and metabolic factors on coronary circulation, we killed the debanded rats and studied coronary hemodynamics under nonworking conditions at two different times after debanding.

\section{Methods}

We used male Wistar rats $6 \mathrm{wk}$ old and banded the ascending aorta. 4 wk after aortic banding some of these rats were debanded. In vivo left ventricular and aortic pressures were measured, and rats were sacrificed 4 wk after banding in the group of banded rats $(n=8)$, and 1 ( $n$ $=7)$ or $4 \mathrm{wk}(n=8)$ after debanding in the debanded groups of rats. Isolated hearts were perfused with modified Krebs-Henseleit buffer and coronary hemodynamics were estimated as in the reports of Lorell et al. (2), Schrader et al. (32), Isoyama et al. (33), and Toma et al. (34). In control rats for each group, sham operations and measurements of in vivo pressures and coronary hemodynamics after sacrifice were performed as in the experimental rats.

Surgical procedures. Each rat was anesthetized with pentobarbital sodium $(50 \mathrm{mg} / \mathrm{kg}$ i.p.) and endotracheal intubation was performed with direct visualization as in the previous study $(35,36)$. The left thorax was opened at the third intercostal space to expose the ascending aorta under artificial ventilation with room air (model 141; Princeton Medical Instruments Inc., Natick, MA). The ascending aorta was dissected free and a surgical nylon thread (3-0) was drawn under the ascending aorta. A rigid tube $(1.4 \mathrm{~mm}$ in o.d.) was placed alongside the ascending aorta. The rigid tube and the ascending aorta were tied tightly together with the thread. The rigid tube was removed rapidly, leaving the ascending aorta constricted to a diameter of 1.4 $\mathrm{mm}$. The lung was inflated with a positive end-expiratory pressure of $\sim 10 \mathrm{~cm} \mathrm{H}_{2} \mathrm{O}$, and the chest was closed with a silk thread. The tracheal tube was removed, and the rats were fed with standard rat chow and water ad lib. for $4 \mathrm{wk}$. In sham-operated rats, the same procedures were repeated, except for aortic banding.

In some of the banded rats, $4 \mathrm{wk}$ after aortic banding the left thorax was opened again under artificial ventilation with room air after intraperitoneal anesthesia and tracheal intubation as described above. Fibrotic tissue around the nylon thread that had been used for aortic banding in the first operation was removed and the nylon thread was cut off. The chest was closed in the same manner as described above. The tracheal tube was removed, and the rats were fed as described above. Sham operations were performed in additional rats to serve as 
their respective control groups. In these procedures, we were able to remove the nylon thread in 15 of 20 rats, and all rats from which the threads were removed survived.

In vivo pressure measurements. Each rat was anesthetized intraperitoneally and tracheal intubation was performed after tracheotomy. Under controlled ventilation with room air, the neck region was carefully opened and the right carotid artery was approached. A polyethylene cannula (polyethylene tubing, SP-31, $0.50 \mathrm{~mm}$ i.d., $0.80 \mathrm{~mm}$ o.d.; Natsume Instrument Co., Ltd., Tokyo, Japan) was inserted into the right carotid artery to measure aortic pressure as in the previous study $(35,36)$. To estimate left ventricular peak systolic and end-diastolic pressures, and the peak-to-peak pressure difference between the left ventricle and the aorta, a left thoracotomy was performed at the fourth intercostal space. The left ventricular cavity was approached from the left ventricular base with a 19-gauge needle, through which left ventricular pressure was measured using a strain gauge transducer (model TP-300T; Nihon Kohden Co., Ltd., Tokyo). Zero pressure reference was taken at the midlevel of the heart. Recordings of phasic and mean pressures were continuously displayed on a multichannel recorder (type 8S, Rectigraph; San-ei Instrument Co., Ltd., Tokyo). Particular care was taken so that the epicardial large coronary arteries were not damaged by needle puncture.

Frequency response of the pressure measurement system was estimated by the pop method. The damping coefficient and undamped natural frequency were 0.56 and $41 \mathrm{~Hz}$, respectively.

Perfusion technique. After in vivo pressure measurements, the pericardium was opened and the heart quickly isolated. A perfusion cannula was inserted into the ascending aorta (at the proximal portion of the thread which was used for aortic banding in the group of banded rats) and positioned immediately above the aortic valve. The heart was mounted on a perfusion apparatus, and the coronary arteries were perfused via the aortic root. The perfusion system consists of a rotary pump and two reservoirs; one of them was pressurized with a gas tank of $5 \% \mathrm{CO}_{2}-95 \% \mathrm{O}_{2}$ and a pressure regulator (pressure regulator type 70; Bellofram, Burlington, MA). The perfusate consisted of modified Krebs-Henseleit buffer: $118 \mathrm{mM} \mathrm{NaCl}, 4.7 \mathrm{mM} \mathrm{KCl}, 2.0 \mathrm{mM} \mathrm{CaCl}_{2}$, $1.2 \mathrm{mM} \mathrm{KH}_{2} \mathrm{PO}_{4}, 1.2 \mathrm{mM} \mathrm{MgSO}{ }_{4}, 25 \mathrm{mM} \mathrm{NaHCO}{ }_{3}, 0.4 \mathrm{mM}$ $\mathrm{Na}_{2}$ EDTA, $5.5 \mathrm{mM}$ dextrose, and $1.0 \mathrm{mM} \mathrm{Na}$ lactate as substrate. Lactic acid was neutralized with $\mathrm{NaOH}$ before being added to the buffer. The oxygenated perfusate was equilibrated with a $5 \% \mathrm{CO}_{2}-95 \%$ $\mathrm{O}_{2}$ gas mixture such that the perfusate $\mathrm{PO}_{2}$ was $\sim 550 \mathrm{mmHg}$. A drainage cannula was inserted into the left ventricular cavity through a left atrial incision to vent the Thebesian flow. To ascertain the stability of the isolated heart, a latex balloon which was slightly larger than the left ventricular cavity was inserted into the left ventricular cavity, and tied at the atrioventricular groove to maintain the heart isovolumic or empty by balloon collapse. The temperature of the perfusate was maintained at $37^{\circ} \mathrm{C}$. Heart rate was kept constant at $300 \mathrm{bpm}$ by right ventricular pacing, using an electrical stimulator (electrical stimulator SEN 7103; Nihon Kohden Co., Ltd.).

Coronary perfusion pressure was measured with a pressure transducer from the side arm of the perfusion cannula. Left ventricular pressure was measured through a polyethylene tube which was connected to the intraventricular balloon. Zero pressure reference was taken at the midlevel of the heart. Mean coronary flow rate was measured using an electromagnetic flow meter (electromagnetic flowmeter, model MFV-1200; Nihon Kohden Co., Ltd.) which was positioned in the perfusion line. Calibration was performed by timed volume sampling.

Protocol to estimate coronary hemodynamics. At a coronary perfusion pressure of $100 \mathrm{mmHg}$ the left ventricular end-diastolic pressure was set to $10 \mathrm{mmHg}$ by adjusting the left ventricular balloon volume during stabilization. After measurement of left ventricular pressure, coronary perfusion pressure and flow rate, the balloon was collapsed. After the coronary flow rate reached steady state, coronary perfusion pressure was stepwisely reduced to $0 \mathrm{mmHg}$ for $1 \mathrm{~min}$ and returned to $100 \mathrm{mmHg}$ to measure peak mean coronary flow rate under the condition of maximal vasodilation. After coronary flow rate had returned to the baseline at $100 \mathrm{mmHg}$ of coronary perfusion pressure, the coronary perfusion pressure was set to $150 \mathrm{mmHg}$. We measured baseline and peak mean coronary flow rate after $1 \mathrm{~min}$ of ischemia at 150 $\mathrm{mmHg}$ of coronary perfusion pressure. The left ventricular balloon was then inflated again to set the left ventricular end-diastolic pressure at $10 \mathrm{mmHg}$. Left ventricular peak systolic and end-diastolic pressures and coronary flow rate returned to baseline $3 \mathrm{~min}$ after ischemia. Coronary flow rate was expressed both as absolute value $(\mathrm{ml} / \mathrm{min})$ and as the ratio of coronary flow rate/left ventricular wet weight $(\mathrm{ml} / \mathrm{min}$ per $\mathrm{g}$ ).

Peak systolic left ventricular pressures at an end-diastolic pressure of $10 \mathrm{mmHg}$ were as follows. In all hearts of the three sham-operated groups, the values were $108 \pm 8$ and $136 \pm 9 \mathrm{mmHg}$ at 100 and 150 mmHg of coronary perfusion pressure, respectively. In hearts of the banded group, the values tended to be greater at each coronary perfusion pressure, compared to those in hearts of the sham-operated groups $(145 \pm 18$ and $159 \pm 22 \mathrm{mmHg}$ ). $1 \mathrm{wk}$ after debanding the values were similar to those in hearts of the banded group (132 \pm 17 and $162 \pm 20$ $\mathrm{mmHg}$ ), but decreased to levels similar to those in hearts of the shamoperated groups $4 \mathrm{wk}$ after debanding $(120 \pm 10$ and $132 \pm 12 \mathrm{mmHg})$.

At the conclusion of the experiment, the heart was stripped of fat and atria, and divided into a right ventricular free wall portion and a left ventricular-septal portion. After determining the left and right ventricular wet weights, they were dried to a constant weight at $70^{\circ} \mathrm{C}$ $(72 \mathrm{~h})$. The ratios of dry weight/wet weight in the left and right ventricles were obtained.

Statistical analysis. Variables measured are expressed as mean \pm SEM. The statistical significance of differences in mean values from two groups of sham-operated and experimental rats or from banded rats and debanded groups of rats were assessed by the unpaired Student's $t$ test. The Bonferroni correction was applied for multiple comparisons to reduce the possibility of chance significance.

\section{Results}

Fig. 1 shows in vivo pressure tracings obtained 4 wk after banding and 4 wk after debanding in rats of banded and debanded groups, respectively. In a representative banded rat shown here, peak systolic left ventricular and aortic pressures were 163 and $77 \mathrm{mmHg}$, respectively. In a representative debanded rat, peak systolic left ventricular pressure was 93 $\mathrm{mmHg}$ and the peak-to-peak pressure difference between the

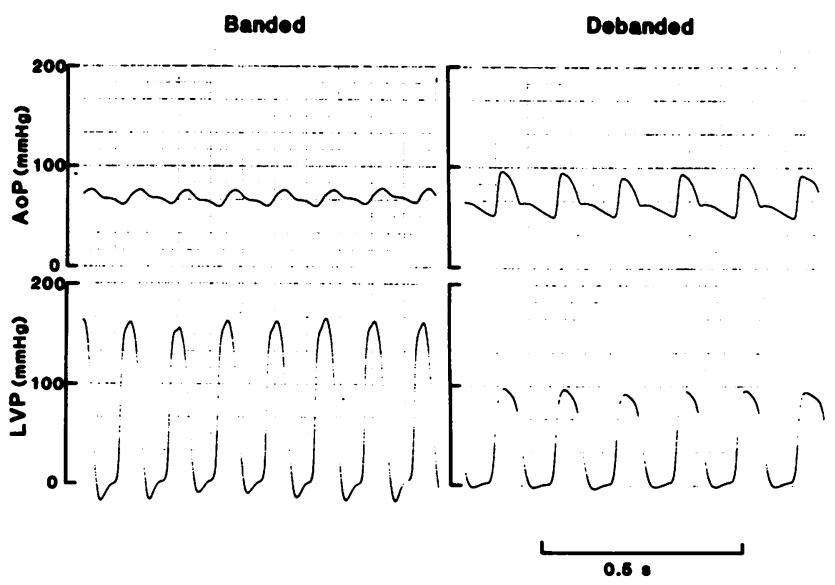

Figure 1 . In vivo left ventricular and aortic pressure tracings in a rat with ascending aortic banding and in a rat 4 wk after debanding. LVP, left ventricular pressure; AoP, aortic pressure measured with a right carotid artery cannula. 
left ventricle and the aorta was only $6 \mathrm{mmHg}$. The left ventricular end-diastolic pressure was $3 \mathrm{mmHg}$ in both the banded and debanded rats.

Fig. 2 and Table I show in vivo left ventricular and aortic pressures and heart rate in the three groups of rats. Peak systolic left ventricular pressure was significantly higher in the banded rats than in the sham-operated rats. In the debanded rats, peak systolic left ventricular pressure measured 1 or 4 wk after debanding decreased significantly, and there was no difference between the sham-operated and debanded rats (Table I and Fig. 2). The peak-to-peak pressure difference between the left ventricle and the aorta in the banded rats was $87 \pm 11$ $\mathrm{mmHg}(P<0.01)$. After debanding the peak-to-peak pressure difference measured 1 or 4 wk after debanding decreased to $12 \pm 8$ and to $11 \pm 2 \mathrm{mmHg}$, respectively; there was no statistical significance in the pressure difference between the sham-operated control and experimental rats (Fig. 2). There was no significant difference in heart rate, left ventricular end-diastolic pressure, systolic, diastolic or mean aortic pressure between the sham-operated and experimental rats in any group ( $\mathrm{Ta}-$ ble I).

As summarized in Table II, body weight at sacrifice did not differ significantly between the sham-operated and experimental rats in the banded or debanded groups. The ratio of left ventricular dry weight/body weight was significantly greater in the banded rats than in the sham-operated rats $(P<0.01)$ (Table II and Fig. 3). 1 wk after debanding, the ratio decreased significantly $(P<0.01)$, but it was still greater than that in the sham-operated rats $(P<0.01) .4 \mathrm{wk}$ after debanding, the ratio decreased further and there was no significant difference between the sham-operated controls and debanded rats. The increase in the degree of cardiac hypertrophy in the banded rats was $48 \pm 5 \%$ above that of control rats; the value decreased to $23 \pm 6 \%$ by 1 wk $(P<0.01)$ and to $11 \pm 6 \% 4$ wk after debanding $(P<0.01)$, as shown in Fig. 3. On the other hand, there was no significant difference in the ratio of right ventricular dry weight/body weight between the sham-operated and experimental rats. Water content of the left and right ventricles was similar in the sham-operated and experimental rats, and among the three groups of rats (Table II).

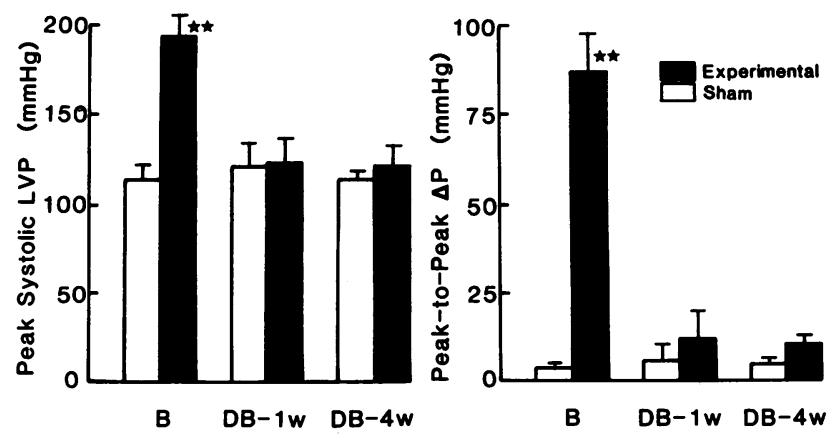

Figure 2. Peak systolic left ventricular pressure and peak-to-peak pressure difference between the left ventricle and the aorta in the three groups of rats. Values are mean \pm SEM. Abbreviations: $B$, banded group of rats; DB-1w, debanded group of rats-1 wk after debanding; DB-4w, debanded group of rats- 4 wk after debanding; $\Delta \mathrm{P}$, pressure difference between the left ventricle and the aorta. ${ }^{*} P$ $<0.05,{ }^{* *} P<0.01$, statistical significance of differences between mean values in sham-operated and experimental rats.
Fig. 4 shows the absolute value of coronary flow rate and the value per myocardial tissue weight at the coronary perfusion pressure of $100 \mathrm{mmHg}$ in the three groups of rats. In the banded group the coronary flow rate decreased under both the control perfusion $(P<0.05)(A)$ and maximal vasodilation $(P$ $<0.01)(B)$, compared with their repective sham-operated controls. In the group of debanded rats, the coronary flow rate increased with time toward the value of the sham-operated controls under both the control perfusion and maximal vasodilation.

The ratio of coronary flow rate/myocardial tissue weight in the banded rats significantly decreased under both control perfusion $(P<0.01, C)$ and maximal vasodilation $(P<0.01$, $D)$. The ratio increased with time, but the value measured 1 wk after debanding remained lower than the value in sham-operated controls under control perfusion $(P<0.01)$ and maximal vasodilation $(P<0.01)$. However, 4 wk after debanding the ratio increased toward the value of sham-operated rats; there was no significant difference between the sham-operated and debanded rats.

Fig. 5 shows the coronary flow rate and the value per myocardial tissue weight at the coronary perfusion pressure of 150 $\mathrm{mmHg}$ in the three groups. There was no significant difference in the coronary flow rate measured under control perfusion between the sham-operated control and experimental rats in any group as shown in panel $A$. Under maximal vasodilation $(B)$, the coronary flow rate was slightly lower in the banded rats than in the sham-operated controls, and tended to increase 4 wk after debanding. However, these changes were not statistically significant.

In the banded rats the ratio of coronary flow rate/myocardial tissue weight was significantly lower under both the control perfusion $(P<0.05, C)$ and maximum vasodilation $(P$ $<0.01, D$ ), compared to sham-operated controls. The value remained lower 1 wk after debanding under both the control perfusion $(P<0.01)$ and maximal vasodilation $(P<0.05)$, compared to sham-operated controls. 4 wk after debanding, there was no significant difference between the sham-operated control and experimental rats.

Fig. 6 shows the changes in coronary flow rate during maximal vasodilation (top) and the flow rate per myocardial tissue weight expressed as percentage of their respective shamoperated controls (bottom) at 100 or $150 \mathrm{mmHg}$ of coronary perfusion pressure in the three groups of rats. Coronary flow rate at the coronary perfusion pressure of $100 \mathrm{mmHg}$ decreased to $72 \pm 5 \%$ of the control value in the banded rats. After debanding the coronary flow had increased to $94 \pm 10 \%$ at 1 wk $(P<0.05)$, and to $108 \pm 9$ at 4 wk $(P<0.01)$. In the banded rats, the coronary flow rate expressed as percentage of shamoperated controls was greater at $150 \mathrm{mmHg}$ than at 100 $\mathrm{mmHg}$ of coronary perfusion pressure $(85 \pm 5$ vs. $72 \pm 5 \%, P$ $<0.05$ ). At the coronary perfusion pressure of $150 \mathrm{mmHg}$ the coronary flow rate increased with time as was observed with the coronary perfusion pressure of $100 \mathrm{mmHg}$.

The ratio, coronary flow rate during maximal vasodilation/myocardial tissue weight, decreased to $53 \pm 4 \%$ at 100 $\mathrm{mmHg}$ and to $61 \pm 4 \%$ at $150 \mathrm{mmHg}$ of coronary perfusion pressure. $1 \mathrm{wk}$ after debanding the ratio did not increase at either 100 or $150 \mathrm{mmHg}$ of coronary perfusion pressure; however, at 4 wk the ratio had significantly increased for both the levels of coronary perfusion pressure $(95 \pm 8 \%$ at $100 \mathrm{mmHg}, P$ $<0.01$ and $100 \pm 10 \%$ at $150 \mathrm{mmHg}, P<0.01$ ). 
Table I. In Vivo Left Ventricular and Aortic Pressures, and Heart Rate in the Three Groups of Rats

\begin{tabular}{|c|c|c|c|c|c|c|}
\hline & Sham-B & B & Sham-lw & DB-1w & Sham-4w & DB-4w \\
\hline & $n 7$ & 8 & 5 & 7 & 6 & 8 \\
\hline HR (bpm) & $401 \pm 24$ & $440 \pm 15$ & $387 \pm 21$ & $401 \pm 21$ & $395 \pm 20$ & $418 \pm 15$ \\
\hline Syst LVP $(m m H g)$ & $114 \pm 9$ & $194 \pm 13^{* *}$ & $121 \pm 15$ & $124 \pm 15$ & $114 \pm 5$ & $122 \pm 12$ \\
\hline LVEDP $(m m H g)$ & $3 \pm 0.6$ & $3 \pm 0.7$ & $2 \pm 0.7$ & $3 \pm 0.7$ & $4 \pm 0.7$ & $3 \pm 0.2$ \\
\hline Syst AoP $(m m H g)$ & $110 \pm 9$ & $105 \pm 7$ & $114 \pm 11$ & $112 \pm 10$ & $108 \pm 5$ & $112 \pm 9$ \\
\hline Diast AoP $(\mathrm{mmHg})$ & $83 \pm 11$ & $78 \pm 4$ & $89 \pm 10$ & $78 \pm 10$ & $86 \pm 7$ & $83 \pm 8$ \\
\hline Mean AoP $(\mathrm{mmHg})$ & $92 \pm 11$ & $86 \pm 4$ & $100 \pm 11$ & $90 \pm 10$ & $97 \pm 6$ & $92 \pm 9$ \\
\hline
\end{tabular}

Values are mean \pm SEM. Abbreviations: Sham-B, sham-operated controls for banded group of rats; B, banded group of rats; Sham-1w, shamoperated controls for the debanded group of rats -1 wk after debanding; DB-1w, debanded group of rats-1 wk after debanding; Sham-4w, sham-operated controls for debanded group of rats-4 wk after debanding, DB-4w, debanded group of rats-4 wk after debanding; HR, heart rate; Syst LVP, peak systolic left ventricular pressure; LVEDP, left ventricular end-diastolic pressure; Syst and Diast AoP, systolic and diastolic aortic pressure. ${ }^{*} P<0.05,{ }^{* *} P<0.01$, statistical significance of differences between mean values in sham-operated and experimental rats.

\section{Discussion}

Our new major findings are as follows: (a) decreased coronary flow rate in hypertrophied hearts produced by pressure overload increased toward normal with time after normalization of the elevated pressure, but the ratio of coronary flow rate during maximal vasodilation per unit myocardial mass remained significantly lower than control values in the early phase of regression process, and $(b)$ decreased coronary flow rate or decreased ratio was completely reversible in the late phase, as in cardiac muscle hypertrophy estimated by myocardial tissue weight. These findings provide useful insights for understanding the pathophysiology of coronary vascular abnormalities in the heart with pressure overload and after its normalization.

In this study, we used an experimental model of pressure overload by aortic banding rather than renal hypertension or a genetic model such as the spontaneous hypertensive rat, to study changes in coronary circulation and myocardial hypertrophy after removing the stimulus for the following reasons. First, the onset of pressure load and normalization of the elevated pressure would be slower in the renal hypertension model. Second, neurohumoral factors may affect myocardial hypertrophy, and pressure may not be the sole determinant of the degree of myocardial hypertrophy and changes in the coro- nary vascular system in a genetic or renal hypertension model $(19,28)$. In fact, we were able to normalize left ventricular pressure after debanding, although minimal pressure difference between the left ventricle and the aorta was observed 1 or $4 \mathrm{wk}$ after debanding. Furthermore, we could observe the regression of myocardial hypertrophy 1 or $4 \mathrm{wk}$ after debanding as reported by Cutilleta et al. (26).

Our methods for estimation of coronary hemodynamics have advantages, compared with the microsphere method in situ. In situ, high left ventricular pressure per se may contribute to changes in coronary vascular hemodynamics through high extravascular tissue pressure, especially the inner layer of the left ventricular wall in pressure-induced hypertrophied hearts. Also, increased myocardial oxygen demand by high left ventricular pressure or wall stress may change the coronary vascular resistance. Furthermore, measurement of coronary flow in situ may be modified by the aortic pressure level $(3,7$, $15)$, i.e., by higher coronary perfusion pressure in a systemic hypertension or aortic banding model than in control subjects, and by the difference between left ventricular pressure and coronary perfusion pressure in animals or patients with aortic valvular stenosis. Even estimation of coronary vascular resistance at one point of coronary perfusion pressure would not be sufficient to determine the coronary circulation abnormalities

Table II. Body Weights, Heart Weights, and Water Contents in the Three Groups of Rats

\begin{tabular}{|c|c|c|c|c|c|c|}
\hline & Sham-B & B & Sham-1w & DB-1w & Sham-4w & DB-4w \\
\hline & n 7 & 8 & 7 & 7 & 8 & 8 \\
\hline BW $(g)$ & $376 \pm 6$ & $355 \pm 22$ & $337 \pm 18$ & $372 \pm 10$ & $371 \pm 20$ & $401 \pm 15$ \\
\hline LV wet W ( $m g$ ) & $849 \pm 23$ & $1148 \pm 81$ & $718 \pm 47$ & $1005 \pm 49$ & $832 \pm 46$ & $978 \pm 81$ \\
\hline $\mathrm{RV}$ wet $\mathrm{W}(\boldsymbol{m g})$ & $227 \pm 11$ & $196 \pm 18$ & $180 \pm 15$ & $211 \pm 6$ & $202 \pm 19$ & $218 \pm 22$ \\
\hline LV dry $\mathbf{W}(m g)$ & $151 \pm 2$ & $211 \pm 14$ & $135 \pm 7$ & $184 \pm 8$ & $149 \pm 8$ & $180 \pm 12$ \\
\hline RV dry W $(m g)$ & $40 \pm 2$ & $38 \pm 2$ & $35 \pm 3$ & $40 \pm 2$ & $34 \pm 1$ & $40 \pm 3$ \\
\hline LV dry W/BW & $0.403 \pm 0.006$ & $0.597 \pm 0.021^{* *}$ & $0.399 \pm 0.010$ & $0.493 \pm 0.024^{* *}$ & $0.404 \pm 0.012$ & $0.449 \pm 0.026$ \\
\hline RV dry W/BW & $0.108 \pm 0.006$ & $0.108 \pm 0.006$ & $0.104 \pm 0.008$ & $0.108 \pm 0.006$ & $0.094 \pm 0.005$ & $0.100 \pm 0.006$ \\
\hline LV Water content (\%) & $82.1 \pm 0.3$ & $81.5 \pm 0.4$ & $81.0 \pm 0.8$ & $81.7 \pm 0.4$ & $81.3 \pm 0.4$ & $81.4 \pm 0.7$ \\
\hline RV Water content $(\%)$ & $82.2 \pm 0.3$ & $80.3 \pm 1.0$ & $80.8 \pm 1.0$ & $81.0 \pm 0.7$ & $81.8 \pm 0.6$ & $81.1 \pm 0.9$ \\
\hline
\end{tabular}

Values are mean \pm SEM. Abbreviations: $B W$, body weight at sacrifice; $L V$, left ventricle; $R V$, right ventricle. Other abbreviations are the same as in Table I. ${ }^{*} P<0.05,{ }^{* *} P<0.01$, statistical significance of differences between mean values in sham-operated and experimental rats. 

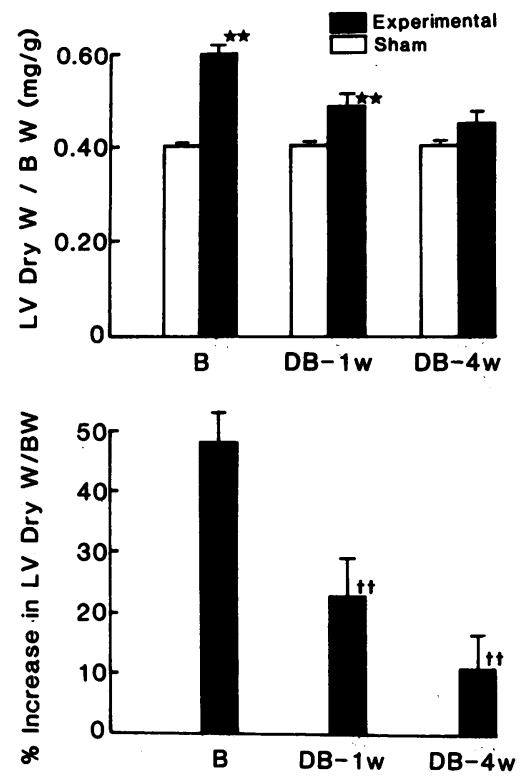

Figure 3. The ratio of left ventricular dry weight per body weight and percent increase in the ratio in the three groups of rats. Values are mean \pm SEM. Abbreviations: $\mathrm{LVW}$, left ventricular weight; BW, body weight. Other abbreviations and symbols are the same as in Fig. 2.

in hypertrophied hearts (19), since the coronary pressure-flow relationship in hypertrophied hearts may differ from that in normal subjects $(37,38)$ and would not be linear. In this study we estimated changes in coronary hemodynamics under non-
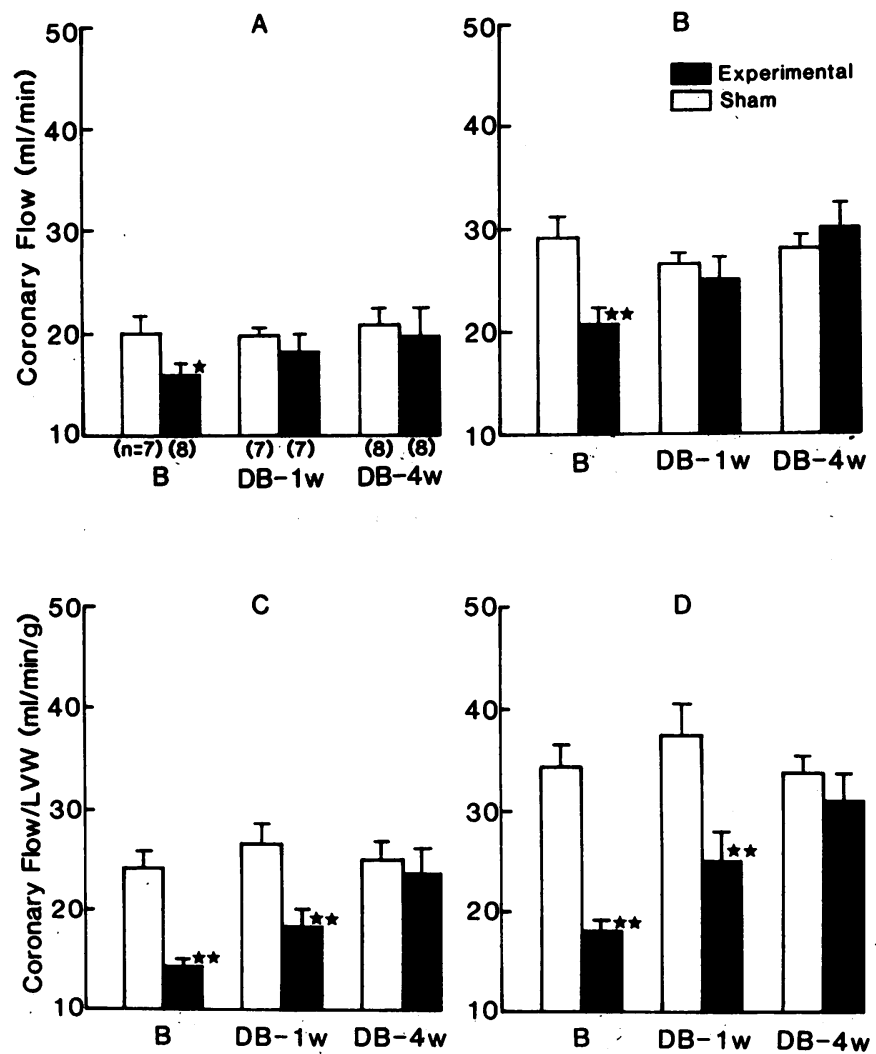

Figure 4. Absolute values of coronary flow rate during control perfusion $(A)$ and during maximal vasodilation after 1-min ischemia $(B)$, and the ratio of coronary flow rate per myocardial tissue weight during control perfusion $(C)$ and during maximal vasodilation $(D)$ at $100 \mathrm{mmHg}$ of coronary perfusion pressure in the three groups of rats. Values are mean \pm SEM. Abbreviations and symbols are the same as in Fig. 2.
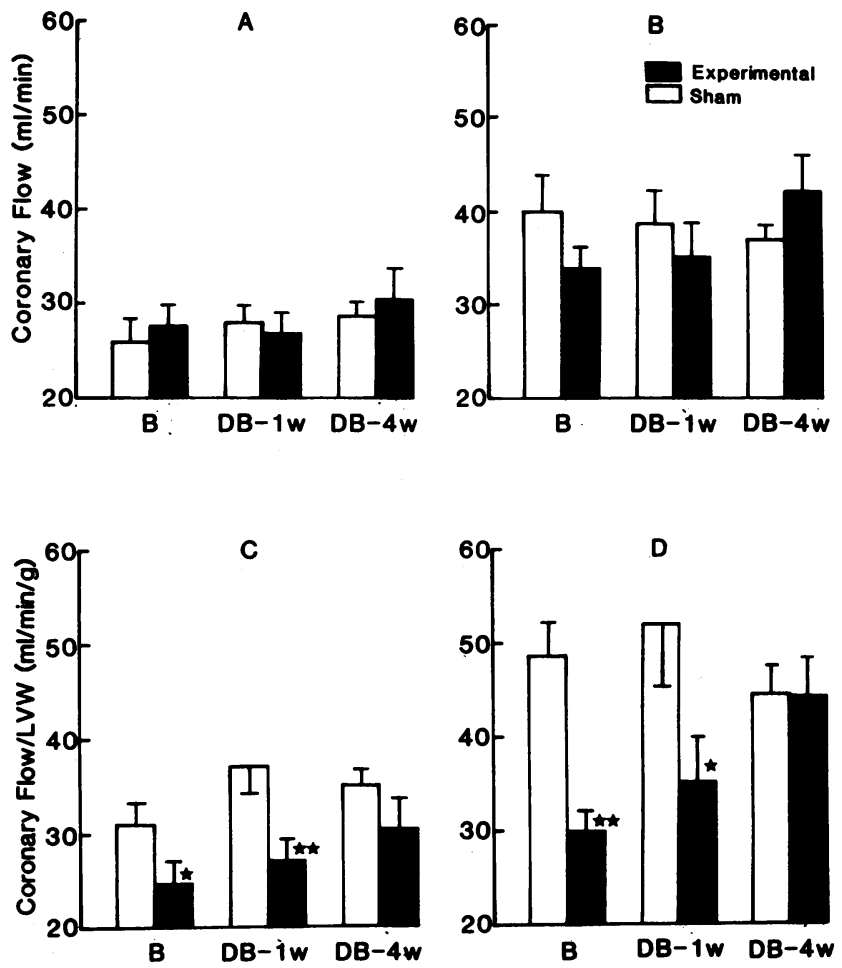

Figure 5. Absolute values of coronary flow rate during control perfusion $(A)$ and during maximal vasodilation after $1-\mathrm{min}$ ischemia $(B)$, and coronary flow rate per myocardial tissue weight ratio during control perfusion $(C)$ and during maximal vasodilation $(D)$ at a coronary perfusion pressure of $150 \mathrm{mmHg}$ in the three groups of rats. Values are mean \pm SEM. Abbreviations and symbols are the same as in Fig. 2.

working conditions to minimize the effects of metabolic and extravascular mechanical factors, and at two different levels of coronary perfusion pressure.

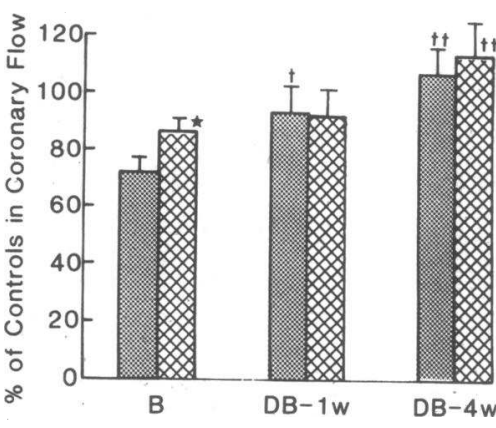

Fure 6. Changes in coronary flow rate during maximal vasodilation and the ratio of coronary flow rate during maximal vasodilation per left ventricular tissue weight at 100 or $150 \mathrm{mmHg}$ of coronary perfusion pressure expressed as percentage of the values in respective

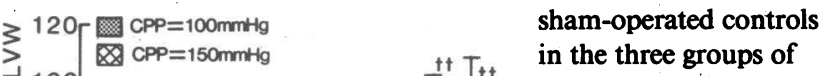

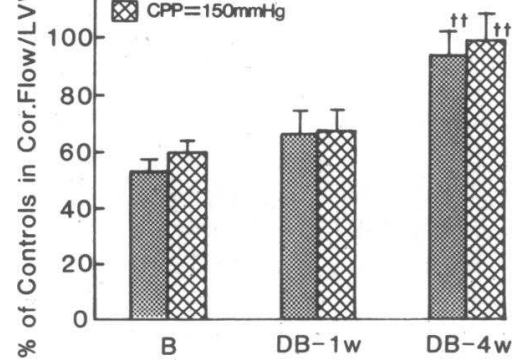
rats. Values are mean \pm SEM. Abbreviations are the same as in Fig. $2 .{ }^{*} P<0.05,{ }^{* *} P<$ 0.01 , statistical signifcance of differences between mean values at 100 and $150 \mathrm{mmHg}$ of coronary perfusion pressure. $\dagger P<0.05$, $\dagger+P<0.01$, statistical significance of differences between mean values in banded and debanded groups of rats. 
As reported by Schrader et al. (32), Toma et al. (35), and Perters et al. (39) we observed a moderate increase in coronary flow rate after brief ischemia, even in the control hearts, compared with observations in human or relatively large animals. However, in small animals such as rats, mice, or guinea pigs the increase in coronary flow rate after brief ischemia is at most $\sim 100 \%$ above baseline even if the coronary hemodynamics are measured in situ. Therefore, the isolated heart preparation which was used in this study is suitable for demonstrating changes in coronary flow rate between hypertrophied and control hearts, and between banded and debanded hearts.

In our study, the absolute values of coronary flow rate at the coronary perfusion pressure of $100 \mathrm{mmHg}$ under control perfusion and maximal vasodilation were significantly lower in banded rats (Fig. 4, $A$ and $B$ ). These findings in hypertrophied hearts show the changes in coronary vasculature per se, since the effects of the extravascular mechanical and metabolic factors were minimized at the same level of coronary perfusion pressure among the banded, debanded and sham-operated control rats. Furthermore, at the higher level of coronary perfusion pressure the absolute value of coronary flow rate under control perfusion or maximal vasodilation did not decrease compared with sham-operated controls (Fig. 5, $A$ and $B$ ). These findings indicate that the pressure-flow relationship in the hypertrophied hearts was different from that in the shamoperated controls. From these observations, the changes in the coronary flow rate in hypertrophied hearts may not have been caused by rarefaction of arterioles or capillaries, as reported by Marcus et al. (20) and Tomanek et al. (40). If rarefaction of coronary vasculature was mainly responsible for the decreased coronary flow in the hypertrophied hearts, coronary flow rate would have decreased at $150 \mathrm{mmHg}$ of coronary perfusion pressure as was observed at $100 \mathrm{mmHg}$ of coronary perfusion pressure. Furthermore, it is unlikely that the normalization in coronary flow rate and the pressure-flow relationship was produced by new growth of coronary vasculature after debanding. If the major stimuli for growth of coronary vasculature are increased systolic pressure or wall stress, increased oxygen demand of the left ventricle or myocytes and/or tissue hypoxia (38), it would be reasonable to assume that all the factors providing the stimulus were decreased by debanding. Therefore, rarefaction or new growth of coronary vasculature may not account for the reversible coronary flow changes in hypertrophied hearts. Tomanek et al. (40) studied vascular hypertrophy of the coronary arterial trees (wall/lumen ratio) in an experimental canine model of renal hypertension and reported that there was no significant difference between hypertrophied and control hearts. From our study, it is not clear whether reversible abnormalities in coronary circulation were caused by morphologic changes in coronary arterial trees such as vascular hypertrophy, physiological changes without morphologic changes, or both.

In the reports of O'Keefe et al. (4) and Wangler et al. (6), coronary vascular resistance per unit muscle mass during maximal vasodilation was increased by coronary arterial hypertension, but not by the presence of myocardial hypertrophy in the right ventricle in an experimental model of aortic banding or in spontaneously hypertensive rats (coronary hypertension without significant right ventricular hypertrophy). In contrast, Alyono et al. (9) studied coronary hemodynamics using an experimental model of left ventricular hypertrophy produced by valvular aortic stenosis and reported that the presence of left ventricular hypertrophy without coronary arte- rial hypertension increased coronary vascular resistance per myocardial mass during maximal vasodilation. Their findings $(4,6,9)$ indicate that the physiological coronary vascular abnormalities do not always coexist with cardiac muscle hypertrophy. Therefore, it is possible that the abnormalities in coronary circulation persist even after complete regression of cardiac muscle hypertrophy produced by pressure overload with coronary arterial hypertension. In our study, the abnormalities in coronary circulation completely normalized at the time when cardiac muscle hypertrophy regressed (from $48 \pm 5$ to $11 \pm 6 \%$ above control values 4 wk after debanding) (Fig. 3). In the early phase of regression process, a significant increase in coronary perfusion during maximal vasodilation per unit muscle mass was not observed in spite of a regression of cardiac muscle hypertrophy by more than $50 \%$ (from $48 \pm 5$ to $23 \pm 6 \%$ ) (Figs. 3 and 6 ). The time course of regression of coronary perfusion per unit muscle mass seemed to be slightly slower than that in cardiac muscle hypertrophy. These findings indicate that the coronary circulation abnormalities in hypertrophied hearts were completely reversible, but coronary perfusion per unit muscle mass was not parallel with cardiac muscle mass in the early phase of the process of regression of cardiac hypertrophy.

In this study, after removing the relatively short-term pressure overload we examined the regression of cardiac muscle hypertrophy and abnormalities in coronary circulation and did not study the effect of duration or severity of pressure load on the regression process. It has been reported that duration or severity of stimulus affects the progression process of coronary vascular changes $(6,39,41,42)$ and cardiac muscle hypertrophy $(23,43)$, and the regression process of cardiac muscle hypertrophy (26). Duration or severity of stimulus may affect the regression process of coronary vascular abnormalities. Also, it may be important for the regression process at what age of the life cycle the stimulus is given, even if duration or severity of the stimulus is the same among the subjects of different ages. Further studies are needed to clarify how and which factors affect the regression process of coronary vascular abnormalities in hypertrophied hearts.

In conclusion, we have provided the first experimental documentation that coronary circulation abnormalities in hypertrophied hearts are completely reversible as in myocardial hypertrophy. Coronary flow rate increased with time, but coronary flow rate during maximal vasodilation per unit cardiac muscle mass remained lower than control values in the early phase of regression process. These experimental findings suggest that coronary circulation abnormalities in hypertrophied hearts are not fixed but are well reversible under some conditions.

\section{Acknowledgments}

This study was partly supported by grant in aid for Scientific Research (No. 63570379) from the Ministry of Education, Science and Culture of Japan.

\section{References}

1. Panidis, J. P., M. N. Kotler, J.-F. Ren, G. S. Minitz, J. Ross, and P. Kalman. 1984. Development and regression of left ventricular hypertrophy. J. Am. Coll. Cardiol. 3:1309-1320.

2. Johnson, L. L., R. R. Sciacca, K. Ellis, M. B. Weiss, and P. J. Cannon. 1978. Reduced left ventricular myocardial blood flow per unit mass in aortic stenosis. Circulation. 57:582-590. 
3. Mueller, T. M., M. L. Marcus, R. E. Kerber, J. A. Young, R. W. Barnes, and F. M. Abboud. 1978. Effect of renal hypertension and left ventricular hypertrophy on the coronary circulation in dogs. Circ. Res. 42:543-549.

4. O'Keefe, D. D., J. I. E. Hoffman, R. Cheitlin, M. J. O'Neill, J. R. Allard, and E. Shapkin. 1978. Coronary blood flow in experimental canine left ventricular hypertrophy. Circ. Res. 43:43-51.

5. Strauer, B.-E. 1979. Ventricular function and coronary hemodynamics in hypertensive heart disease. Am. J. Cardiol. 44:999-1006.

6. Wangler, R. D., K. G. Peters, M. L. Marcus, and R. J. Tomanek. 1982. Effects of duration and severity of arterial hypertension and cardiac hypertrophy on coronary vasodilator reserve. Circ. Res. 51:10-18

7. Bache, R. J., D. Alyono, E. Sublett, and X.-Z. Dai. 1986. Myocardial blood flow in left ventricular hypertrophy developing in young and adult dogs. Am. J. Physiol. 251:H949-H956.

8. Lorell, B. H., L. F. Wexler, S. Momomura, E. O. Weinberg, and C. S. Apstein. 1986. The influence of pressure overload left ventricular hypertrophy on diastolic properties during hypoxia in isovolumically contracting rat hearts. Circ. Res. 58:653-663.

9. Alyono, D., R. W. Anderson, D. G. Parrish, X.-Z. Dai, and R. J. Bache. 1986. Alterations of myocardial blood flow associated with experimental canine left ventricular hypertrophy secondary to valvular aortic stenosis. Circ. Res. 58:47-57.

10. Harrison, D. G., M. S. Florentine, L. A. Brooks, S. M. Cooper, and M. L. Marcus. 1988. The effect of hypertension and left ventricular hypertrophy on the lower range of coronary autoregulation. Circulation. 77:1108-1115.

11. Bache, R. J., T. R. Vrobel, C. E. Arentzen, and W. S. Ring. 1981. Effect of maximal coronary vasodilation on transmural myocardial perfusion during tachycardia in dogs with left ventricular hypertrophy. Circ. Res. 49:742-750.

12. Bache, R. J., T. R. Vrobel, W. S. Ring, R. W. Emery, and R. W. Andersen. 1981. Regional myocardial blood flow during exercise in dogs with chronic left ventricular hypertrophy. Circ. Res. 48:76-87.

13. Breisch, E. A., F. C. White, L. E. Nimmo, and C. M. Bloor. 1986. Cardiac vasculature and flow during pressure-overload hypertrophy. Am. J. Physiol. 251:H1031-H1037.

14. Marcus, M. L., T. M. Mueller, and C. L. Eastham. 1981. Effects of short- and long-term left ventricular hypertrophy on coronary circulation. Am. J. Physiol. 241:H358-H362.

15. Pichard, A. D., R. Gorlin, H. Smith, J. Ambrose, and J. Meller. 1981. Coronary flow studies in patients with left ventricular hypertrophy of the hypertensive type. Am. J. Cardiol. 47:547-554.

16. Marcus, M. L., D. B. Doty, L. F. Hiratzka, C. B. Wright, and C. L. Eastham. 1982. Decreased coronary reserve. A mechanism for angina pectoris in patients with aortic stenosis and normal coronary arteries. $N$. Engl. J. Med. 307:1362-1366.

17. Pichard, A. D., H. Smith, J. Holt, J. Meller, and R. Gorlin. 1983. Coronary vascular reserve in left ventricular hypertrophy secondary to chronic aortic regurgitation. Am. J. Cardiol. 51:315-320.

18. Opherk, D., G. Mall, H. Zebe, F. Schwarz, E. Weihe, J. Manthey, and W. Kübler. 1984. Reduction of coronary reserve: a mechanism for angina pectoris in patients with arterial hypertension and normal coronary arteries. Circulation. 69:1-7.

19. Tarazi, R. C., and E. D. Frohlich. 1987. Is reversal of cardiac hypertrophy a desirable goal of antihypertensive therapy? Circulation. 75(Suppl. I):I-113-I-117.

20. Marcus, M. L., D. G. Harrison, W. M. Chilian, S. Koyanagi, T. Inou, R. J. Tomanek, J. B. Martins, C. L. Eastham, and L. F. Hiratzka. 1987. Alterations in the coronary circulation in hypertrophied ventricles. Circulation. 75(Suppl. I):I-19-I-25.

21. Hoffman, J. I. E. 1987. A critical view of coronary reserve. Circulation. 75(Suppl. I):I-6-I-11.

22. Nitenberg, A., J.-M. Foult, I. Antony, F. Blanchet, and M. Rahali. 1988. Coronary flow and resistance reserve in patients with chronic aortic regurgitation, angina pectoris and normal coronary arteries. J. Am. Coll. Cardiol. 11:478-486.

23. Dodge, H. T., M. Frimer, and D. K. Stewart. 1974. Functional evaluation of the hypertrophied heart in man. Circ. Res. 34 \& 35(Suppl II):II-122-II-127.

24. Papadimitriou, J. M., B. E. Hopkins, and R. R. Taylor. 1974. Regression of left ventricular dilation and hypertrophy after removal of volume overload. Circ. Res. 35:127-135.

25. Cooper, G. IV, R. M. Satava, C. E. Harrison, and H. N. Coleman III. 1974. Normal myocardial function and energetics after reversing pressure-overload hypertrophy. Am. J. Physiol. 226:11581165.

26. Cutilletta, A. F., R. T. Dowell, M. Rudnik, R. A. Arcilla, and R. Zak. 1975. Regression of myocardial hypertrophy. I. Experimental model, changes in heart weight, nucleic acids and collagen. J. Mol. Cell. Cardiol. 7:767-781.

27. Sen, S., R. C. Tarazi, and F. M. Bumpus. 1976. Biochemical changes associated with development and reversal of cardiac hypertrophy in spontaneously hypertensive rats. Cardiovasc. Res. 10:254261.

28. Sen, S., R. C. Tarazi, and F. M. Bumpus. 1977. Cardiac hypertrophy and antihypertensive therapy. Cardiovasc. Res. 11:427-433.

29. Sen, S., R. C. Tarazi, and F. M. Bumpus. 1981. Reversal of cardiac hypertrophy in renal hypertensive rats: medical vs. surgical therapy. Am. J. Physiol. 240:H408-H412.

30. Perloff, J. K. 1982. Development and regression of increased ventricular mass. Am. J. Cardiol. 50:605-611.

31. Cooper, G., IV, and T. A. Marino. 1984. Complete reversibility of cat right ventricular chronic progressive pressure overload. Circ. Res. 54:323-331.

32. Schrader, J., F. J. Haddy, and E. Gerlach. 1977. Release of adenosine, inosine and hypoxanthine from the isolated guinea pig heart during hypoxia, flow-autoregulation and reactive hyperemia. Pfluegers Arch. Eur. J. Physiol. 369:1-6.

33. Isoyama, S., B. H. Lorell, W. N. Grice, L. F. Wexler, and C. S. Apstein. 1985. Increased diastolic chamber stiffness during simulated angina in isolated hearts. Circulation. 72(Suppl. III):III-72. (Abstr.)

34. Toma, B. S., R. D. Wangler, D. F. DeWitt, and H. V. Sparks, Jr. 1985. Effect of development on coronary vasodilator reserve in the isolated guinea pig heart. Circ. Res. 57:538-544.

35. Isoyama, S., J. Y. Wei, S. Izumo, P. Fort, F. J. Schoen, and W. Grossman. 1987. Effect of age on the development of cardiac hypertrophy produced by aortic constriction in the rat. Circ. Res. 61:337345.

36. Isoyama, S., W. Grossman, and J. Y. Wei. 1988. Effect of age on myocardial adaptation to volume overload in the rat. J. Clin. Invest. 81:1850-1857.

37. Scheel, K. W., and S. E. Williams. 1985. Hypertrophy and coronary and collateral vascularity in dogs with severe chronic anemia. Am. J. Physiol. 249:H1031-H1037.

38. Peters, K. G., R. D. Wangler, R. J. Tomanek, and M. L. Marcus. 1984. Effects of long-term cardiac hypertrophy on coronary vasodilator reserve in SHR rats. Am. J. Cardiol. 54:1342-1348.

39. Scheel, K. W., B. L. Eisenstein, and L. A. Ingram. 1984. Coronary, collateral, and perfusion territory responses to aortic banding. Am. J. Physiol. 246:H768-H775.

40. Tomanek, R. J., P. J. Palmer, G. L. Peiffer, K. L. Schreiber, C. L. Eastham, and M. L. Marcus. 1986. Morphometry of canine coronary arteries, arterioles, and capillaries during hypertension and left ventricular hypertrophy. Circ. Res. 58:38-46.

41. Tomanek, R. J., K. A. Schalk, T. Nadle, and D. G. Harrison. 1987. Coronary vascular growth in dogs with long-term hypertension. Circulation. 76(Suppl. IV):IV-328. (Abstr.)

42. Engelman, G. L., J. C. Vitullo, and R. G. Gerrity. 1987. Morphometric analysis of cardiac hypertrophy during development, maturation, and senescence in spontaneously hypertensive rats. Circ. Res. 60:487-494.

43. Olivetti, G., R. Ricci, and P. Anversa. 1987. Hyperplasia of myocyte nuclei in long-term cardiac hypertrophy in rats. J. Clin. Invest. 80:1818-1821. 